\title{
Health Related Reforms as a Predictor of Change of Behavior Among Inmates. A Study of Correctional Facilities in Kenya
}

\author{
Solomon Gikundi Koome ${ }^{1} \quad$ Charles Wafula ${ }^{2} \quad$ Raphael Nyonje ${ }^{2}$ \\ 1.Correctional Officer State Department of Correction Service. P.O Box 30195-00100 Nairobi- Kenya \\ 2.School of Open and E-learning, University of Nairobi P.O Box 30197-00100 Nairobi- Kenya
}

\begin{abstract}
This study focused on health related reforms on change of behavior of inmates in correctional facilities in Kenya. The study was based on the following objective; to examine how health related reforms influences change of behavior of inmates in correctional facilities in Kenya. The study tested one alternate hypothesis on whether the relationship among the study variable was significant. The target population was 10,111 individuals spread as 2,953 prison officers and 7,158 inmates. From this population a sample was drawn using a formula totaling to 385 individuals who were picked for the study. The sample was spread accordingly as 112 prison officers and 273 inmates. Purposive sampling was used to select officers' in-charge and their deputies. Twelve prison officers on duty were picked randomly from duty roster using prime numbers for focus group discussion from each prison. A separate list of long term prisoners was compiled from the records of capital offence and other offences who have served jail term of over ten years or have been in remand for ten years and then the sample size was picked in proportionate to the population in each prison institution from the list. Primary data was gathered by a semi-structured questionnaire, an interview schedule and focus group discussion from the target population. Secondary data was gathered through analyzing relevant documents. The study incorporated quantitative and qualitative approaches to process, analyze, and interpret the data. The SPSS was applied to run analyses for percentages, frequency distributions, and measures of central tendency, where applicable. Descriptive statistics were computed for variable using frequencies, percentages, mean and standard deviation. Statistical tools used for inferential statistic were person's product moment correlation(r), simple regression, multiple regression and step wise regression $\left(\mathrm{R}^{2)}\right.$. T-tests were used to test hypothesis in the study the results revealed that $\mathrm{r}=0.429, \mathrm{t}=5.189$, at $\mathrm{p}=0.000<0.05 . \mathrm{H}_{1}$ was confirmed and concluded that health reforms had a statistically significant influence on change of behavior of inmates in correctional facilities in Kenya. Findings of the study are expected to assist government policy makers regarding formulation and implementation of the prison reforms. The serving inmates may benefit from the improved prison environment which will be aligned with market preference and whose content may benefit the community.
\end{abstract}

Keys terms : Health related reforms, change of behavior, inmates, correctional facilities

DOI: $10.7176 / \mathrm{JLPG} / 91-13$

Publication date: November $30^{\text {th }} 2019$

\subsection{INTRODUCTION}

Since the conception of the modem state, prison reform has been an issue raising national and international concern. Prisons worldwide are faced with the problem of adjusting their attitude and adapting a proactive system in order to attain their goals and objectives (Prison handbook, 1997). Prisons serve a valuable role in society, by providing a rehabilitative environment for those who break the rule of law in an effort to better them as people. They encourage peace and allow for reform (Adler and Longhurst, 1994). Prison reform in Germany is geared towards deterrence, reformation, and the protection of society. These factors are emphasized differently in different countries. Emphasis has been laid chiefly upon punishment and the protection of society. Empowerment of officers through education is manifested through numerous libraries found in most prisons (Anderson and Overy, 2010).

While people living behind bars are entitled to a right to access of standard healthcare comparable to that accessible to those living outside the prisons. The "principle of equivalence" is among those enjoying extensive consensus amongst human rights instruments and international health organisations. Amidst severe health problems evidenced in prisons globally, the state has a legal obligation of safeguarding lives and health of people it confines. This notwithstanding, prison healthcare standards compared to that prevailing in the community in most cases fail to meet public health needs and human rights obligations (Rick, 2006). The contest concerning reform in healthcare contains problems of sustainability, fairness, right to access healthcare, quality of healthcare offered as well as government spending on healthcare (Helen and Deborah, 2011). As postulated by Toon (2005) efficiency in macro-economic links to the share of resources allocated and channelled to healthcare in aggregate and the benefits that accrue in terms of individual or household health outcomes. General argument is that countries that direct more spending on healthcare and maintain same outcomes are less efficient while those that spend less on healthcare and post better results are termed to be more efficient.

Congested prisons unavoidably lead to exposure of inmates to improper health conditions that consequently 
spread epidemics hence causing death and diseases such as tuberculosis and HIV/AIDS (Achu, Owan and Ekok, 2013). As largely supported by various healthcare systems across the globe, numerous diverse pathways a nation can embrace when considering healthcare reform can be explored. One major component of healthcare improvement is a reduction of healthcare abuse and fraud by the participants. In the United State of America and the European Union, an estimation of 10 per cent of total healthcare expenditures and transactions could possibly be fraudulent (Terry, 2011).

A major challenge that faces the healthcare system across the globe is the comparative lack of funding by public sector to cover the cost of medical services that are already a responsibility of the state, to guarantee quality, affordable or free medical services to the whole population. Toon (2005) argued that it's not meaningful to reform healthcare on piecemeal basis. Toon further extended that pilot initiatives as well as model practices in an area or system may lead to antagonism rather than becoming catalysts for developmental change. Catherine, Helen and Brian (2009) put forward that consultancies for development and funding organizations should be well harmonized and work in numerous countries. By doing this, they create an enabling evidence based experience and develop tools that can be used across several countries. . Although the political will to undertake serious reform is lacking in many countries, some countries are yet ready to move to a healthcare service backed by democratic values. Fein (1994) suggests that the support of long-term development partnerships and educational programmes can encourage local medical experts who could help push for open to change

Prison surroundings consist of institutional areas (prisoners, staff and visitors) and specialized residential quarters for housing prison staff and families living within the institution. Water, sanitation and hygiene (WASH) service needs within prisons are huge in relation to general.

In the Kenyan prisons the medical officers attached to prison institutions should be an independent professional who should maintain a primary doctor-patient relationship with the male and female prisoners. Medical confidentiality should be respected and no medical officer should be involved in any way in the punishment of prisoners but can certify them as medically fit to undergo the punishment they have been prescribed for (IED, 2004)

The detention centres is a component of criminal justice system entrusted institutionally to imprison, reform or rehabilitate lawbreakers and make sure that they abide by the principal society values on discharge. Convicts are therefore expected to access training, proper guidance and counseling and enabled to resocialize as better citizens (Odigha, 2004). Prison welfare officers have a general responsibility of staff and prisoner welfare who theoretically support the process of rehabilitation but in the Kenyan scenario they are too few, poorly equipped and trained to facilitate post release planning and prisoners contact with families and communities (Oketch, 2009). There is therefore a need to review their roles and functions towards rehabilitation and reintegration of offenders and need to recruit more welfare officers and equip them with skills on behavior modification techniques, counseling, and awareness of special needs of offenders. Pre-release planning and management of offenders' re-entry in the society as far as receiving the skills they require can be facilitated by the welfare officers (IED, 2004).

In an open door policy prison, the dream of enlightened prison that embodies five core virtues: hope, merit recognition, individuality, growth, and community would be achieved. In the absence of these attitudes that symbolize characteristics of current-day institutions- persistent of the "endarkened" prison, manifested by despair, isolation, stasis, recognition of demerits and classification will rule. Long serving prison officers often have substantial impact on the staff culture, and among staff there are also informal codes hence similarities in the staff and the inmates' cultures. This leads to distancing and an objectifying approach in relation to prisoners. In the two cultures, stereotypical social representations of 'the other' is created, which have undesirable impression on the possibilities for working out rehabilitation and hinders reporting of incidences on personal health, drug abuse, and planned jail breaks before they get out of hands (Odd, 2005).

Kenya Prisons Service functions are to contain and keep offenders in safely in custody, rehabilitate and reform offenders to control recidivism, containment to detect and prevent escape and jail break and promote prisoner's opportunities for social re-integration. Thus, the department has over the years implemented various models of rehabilitation and reintegration of offender. Planned interventions that endeavor to change the offender's cognitive processes, attitudes, personality or mental health is very essential. Broadly, rehabilitation refer to social relations with others, vocational skills, education and employment, which can be realized by inmates attending and completing various tests (Oketch, 2009). The intent of the intervention is to make the prisoners less likely to break the law in future or basically to reduce recidivism (Law, 2004). Maltz (1981) put forward that reintegration is the whole process through which an individual is reintroduced to the community with an aim of assisting the person to live in a law- should focus at promoting personal responsibility and providing prisoners with practical benefits to succeed in lawful occupation (KNCHR, 2010).

In Africa prison conditions are extremely bad, characterized by overcrowding, inadequate health and sanitation infrastructure, inexistence rehabilitation programme, insufficient food in terms of quality and quantity (KNCHR, 2003). According to Gaes (2008) the aim of any prison institution is to prevent and cure crime as such, 
there is no internationally recognized or accepted model of a prison system. The style of imprisonment is closely related to the culture and history of a country. The question of whether imprisonment does reform, correct, rehabilitate or reintegratecriminals is a question on which world authorities have not agreed (Becker, 2003). Although some offenders have been rehabilitated through imprisonment, what is known worldwide is that most prisons harden the offenders. What a sociologist can confirm is that some of the prison sentences contribute to the disintegration of some of the offenders' families and to the delinquency of their dependent minors thereby increasing the crime rate (Griffiths \& Murdoch, 2009). It's therefore a collective responsibility of every stakeholder to participate in the effort toreform the offenders. In the year 2001, Kenyan prisons reportedly embarked on reforming prison conditions. The right to health is holistic and requires the government to have policies and practices that make it accessible and available. However in Kenya the improvement of prison facilities in relation to health is wanting faced with inadequate participation, insufficient drugs supplies, inadequate facilities and few medical practitioners (KNCHR, 2010). This is contrary to International Covenant on Economic, Social and Cultural Rights which call for availability of functional public health facilities, accessible quality healthcare services without discrimination. Therefore the study sought to seek whether health reforms influences change of behavior of inmates in correctional facilities in Nairobi County, Kenya

\subsection{LITERATURE REVIEW}

In a prison system, it is difficult to provide health services and many concerns about prisoners' health and the quality of healthcare availed to them always come to play. In 2006 health services for prisons across Wales and England were moved to the National Health Service, a move that was adopted by Scotland and Northern Ireland (Smith, 2009). Prison system presents unique challenges for health care practitioners. Effective healthcare comes second, but if good health care is not available to prisoners, they have no substitute as prisons are not considered as nurturing places (Akinyi, 2009). Prisoners by design are a challenging population to treat effectively because their social and health needs are diverse and a large number of prisoners are exposed to poor physical and mental health (Jewkes, 2005). Regular relocations or prisoner transfer and short sentenced prisoners make engagement with healthcare very difficult because of non-continuity with prescribed healthcare and aftercare service especially on specialized treatment.

\subsection{Health Reforms and Change of Behavior of Inmates in Correctional Facilities}

In a prison system, it is difficult to provide health services and many concerns about prisoners' health and the quality of healthcare availed to them always come to play. In 2006 health services for prisons across Wales and England were moved to the National Health Service, a move that was adopted by Scotland and Northern Ireland (Smith, 2009). Prison system presents unique challenges for health care practitioners. Effective healthcare comes second, but if good health care is not available to prisoners, they have no substitute as prisons are not considered as nurturing places (Akinyi, 2009). Prisoners by design are a challenging population to treat effectively because their social and health needs are diverse and a large number of prisoners are exposed to poor physical and mental health (Jewkes, 2005). Regular relocations or prisoner transfer and short sentenced prisoners make engagement with healthcare very difficult because of non-continuity with prescribed healthcare and aftercare service especially on specialized treatment.

In Russian Federation for instance, it was indicated presence of insufficient data to show how spending in prison healthcare has translated into better health results like improved morbidity and mortality, improved productivity or better economic output. Data on hospital outputs and other healthcare providers to allow for control of mixed cases and quality of care is limited in many countries. Therefore, it is complex to assess the distributional impacts or the efficiency of health interventions in penal institutions (Word Bank, 2014).

Exposure to violence, illicit drugs, and victimization is common occurrence in prisons. Prisoners are dependent on prison staff for about every aspect of their survival and hence they have limited ability to control many factors that affect their health directly or indirectly (Wolf et al., 2007). Their diet, accommodation, exercise activities are all beyond the control of prisoners. Another barrier to better healthcare service is that prisons are prone to local politics. Views by the public on the experience of imprisonment and the kind of people found within prisons can be distorted by images painted in the media, which incline to focus on celebrity prisoners, perceptions of prisoners getting undue healthcare privileges, and sentences considered too lenient. As a result, every successful health initiative runs the risk of being regarded as too good for undeserving prisoners (Abuyeka, 2014).

Sarah et al. (2016) in their study found the associations between use of substance and offending. The findings could be useful in provision of multi-focused reforms in prisons aimed at addressing behaviors of offenders. Digital technologies were proposed as practical tools that could provide opportunities to broaden access to interventions via computer-assisted therapy models. Use of digital technologies would also open opportunities for integrated care by availing interventions to community settings and prison as well as follow-up of offender's journey through the criminal justice system. Koech (2009) noted that most of non-violent offenders 
face additional challenges or psychological conditions that can be monitored, checked and managed through mental support services, which could only take up a proportion of costs that imprisoning the person would cost.

\subsection{Theoretical Framework}

The study was anchored on the following theories; Theory of Change developed by Weiss (2000), Theory of Planned Behavior and the Unified Theory ofPunishment by Brooks (2015) the theories are suitable for study because they involve change, program and punishment respectively. Theory of change popularized by Weiss, theory of change describes a set of assumption that explain the stages that result to long term interest goals and the links between activities and outcomes of the program that happen at each phase. A theory of change came about as a result of progressive critical thinking exercises that presents a comprehensive depiction of early and transitional term changes in a certain community that are required to arrive at long-term goal that a community articulates (Weiss, 2000). A theory of change creates a candid image of steps required to attain a goal. It provides a good premise for improving overall evaluation plans and strengthens a community's ability to claim recognition for results that their theory predicted. This is based on the fact that change takes time and therefore, accomplishment may not be recognized as they arise (Connell and Kubisch, 1998). Laying down precisely what you intend to accomplish and how to know whether you are progressing is what would ensure that all the efforts are recognized. Theory of change elucidates the change process through delineating causal linkages in a programme, for instance its short-term, middle-term and long-term results. The acknowledged changes are mapped as the "outcomes pathway"- presenting each outcome in rational relationship to others, as well as sequential flow. Links between outcomes are then explained through statements that show the reason of one outcome being thought as a prerequisite for another (Clark and Taplin, 2012). The improvement of theory of change lies first in distinguishing between actual and desired and outcomes, and second in necessitating stakeholders to model the outcomes they desire before deciding on interventional forms of achieving the required outcomes. Other than outcomes directly related to program, the theory would envisage outcomes that control and those that influence outcomes. This approach could thus aid in avoidance of mapping outcomes that involve expansive shifts in values and behaviour amongst populations which could sometimes difficult to monitor and to attribute to a particular program. Theory of change has identifies three forms of outcomes; influence, impact and leverage, and it could be valuable to identify such outcomes as an approach to focus on change on achievements that are measurable. When using theory of change to guide reform projects, it helps to direct the group's effort on outcomes, which when achieved, can convincingly be attributed to program success (Chen, Mathison and Chen, 2005). The theory of change is also suitable for this study since it involves change particularly in service delivery in prison institutions with specific focus on rehabilitation and reformation, change in attitude, character, behaviour and acquisition of new skills in the short term and long-term.

\subsection{Conceptual Framework Independent Variable}

\section{Dependent Variable}

\begin{tabular}{|c|c|}
\hline HEALTHS REFORMS & $\begin{array}{l}\text { CHANGE OF BEHAVIOR OF } \\
\text { INMATES Increase or decrease in; }\end{array}$ \\
\hline 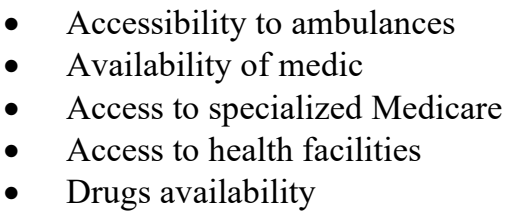 & $\begin{array}{ll}\text { - } & \text { Re-offending } \\
\text { - } & \text { Recidivism } \\
\text { - } & \text { Jail breaksRiots and unruly } \\
& \text { habits } \\
\text { - } & \text { Escapes }\end{array}$ \\
\hline
\end{tabular}

\subsection{RESEARCH METHODOLOGY}

Pragmatism is viewed as a deconstructive paradigm that backs the use of mixed methods in research, a paradigm that circumvents the contention in reality and truth and instead directs focus on 'what is plausible' as the truth concerning the research questions being investigated (Biesta, 2010). The shift of researchers to mixed methods approach showed research designs that adopted "mixing" of quantitative or qualitative approaches when gathering data of a study for instance. This study adopted causal comparative research designs and descriptive cross-sectional survey. The cross-sectional survey design incorporates qualitative and quantitative approaches. The quantitative approach consists of closed-ended questions which prompted information for inferential and descriptive purposes. The qualitative approach with open-ended questions obtained in-depth information to validate inferential and descriptive results (Mwanje, 2001). Across-sectional survey design is argued to be cheaper than longitudinal designs hence they are most appropriate for academic investigators, who in most cases work under strict budget responses rather than ones that reflect their own true opinions (ASA, 1999; Rindfleisch et al., 2008). 
Uma (2003) defines a target population as the actual hypothetical set of events, people or objects which the researcher seeks to generalize the findings. There were 9 prison institutions in Nairobi County, Kenya with a total target population of 10,111 individuals which was spread as 7,158 prisoners and 2,953 prison officers (including 9 officers in-charge, 9 deputy officers in-charge) who formed the population of this study, the sample was gotten from the population using formula below. This decision was based on Cooper and Schindler (2003) regarding the need to purposely include elements in a population in a study, to gather appropriate data for a particular study. They were arranged alphabetically and selected randomly from the list.

Study sought both primary and secondary data. In this regard, three sets of instruments were applied to source requisite data, including a survey questionnaire, an interview schedule and focus group discussion. The application of multiple instruments is important for enhancing validity of data obtained and minimizing the possibility of experiencing interviewer biases. Such biases often arise from non-verbal cues that may influence participants to give misleading responses by reporting positive aspects even where negative aspects are predominant (Jaeger, 1984). The study applied a questionnaire that targets prisoners who were informed about the study and consented to ensure voluntary participation. The instrument contained closed-ended and openended questions structured according to objectives of the study. The questionnaire was structured into two parts. Part 1 details the demographic information, part 2 is divided into six sections $\mathrm{A}, \mathrm{B}$, and $\mathrm{C}$ that captures the two variables of the study.

The instrument captures information that meets the requirements for positivistic paradigm and quantitative approach. The information captured in the simplest variable forms was objective and used to confirm or refute hypothesized influence of health related reforms on change of inmates behavior in correctional facilities. An interview schedule was were applied on officers' in-charge and their deputies who are key informants. The investigator sought consent from officer's in-charge and deputy officer's in-charge and booked appointments which was to be used to develop a data collection itinerary. Participants were given an outline of the topics that were discussed at least a day earlier to ensure constructive deliberations. The tool was sought information on the institutional reforms and change of behavior of inmates. As noted by Touliatos and Compton (1988), evaluating perceptions, views, attitudes, opinions and feelings of individuals can done best through in-depth interview schedules.

Focus discussions are a form of group interviews that capitalize on sharing information between facilitators and participants so as to generate data (Best \& Khan, 2004). Mugenda (2010) view focus group technique as a research method that gathers information through interaction of members of a group on a particular topic that the chosen by the investigator; the interest of investigator guides the focus while the information emanates from group interactions (Morgan, 1997). Though group interviews are frequently used as a convenient and quick way to gather data from numerous people concurrently, focus groups explicitly utilize interactions by groups as part of the method (Creswell, 2008). In this study, FGD guide will target prison officers in different prisons. A group comprising of twelve officers was formed and questions raised from the variables of study were raised and discussed.

Recruited research assistants were guided by the investigator to aid in collecting data. To enhance familiarity with the instrument, the researcher held training sessions with research assistants who also refreshed them on research ethics, enable sharing of essential data sourcing skill especially on the right approach to participants and building rapport with the respondents. Data collection commenced on approval by relevant authorities such as the commissioner general of prison and also acquire authorization to carry on research from the National Commission for Science and Technology (NACOSTI).

Both quantitative and qualitative approaches were applied in processing, analyzing, and interpretation of the data. Quantitative data processing involves coding close-ended data, cleaning, entry, transformation, analyzing and interpretation (Obure, 2002). To generate percentages, frequency distributions, and measures of central tendency, the SPSS programme was used to run analysis. In addition, charts, graphical presentations and tables were produced using Ms-Excel package. Descriptive and multiple regressions were run especially to assess the relationship among independent variable and dependent variable. To test for the hypothesis; $\mathrm{H}_{0} 1$ : There is no significant relationship between health reforms and change of behavior of inmate in correctional facilities in Nairobi County, Kenya. Regression analysis by Alexopoulos (2010) was used.

$$
Y_{1}=a+B X+\varepsilon
$$

Where; $\quad \mathrm{Y} 1=$ change of behavior of inmates in correctional facilities

$a=$ constants $/$ Intercept $\beta=$ Coefficient

$\mathrm{X}=$ health reforms

$\varepsilon=$ Error term

Coefficient $\beta$ denote the change of behavior of inmates in correctional facilities from a change in health reform. Relationship exists if $\beta$ is statistically significant. 


\subsection{FINDINGS}

This chapter presents the study results which have been discussed based on thematic and sub-thematic areas of study objective. Thematic areas are idealized as, health related reforms and change of behavior of inmates in correctional facilities. Both quantitative and qualitative approaches were applied in processing, analyzing, and interpretation of the data. To generate percentages, frequency distributions, and measures of central tendency, the SPSS programme was used to run analysis. In addition, charts, graphical presentations and tables were produced. Descriptive and multiple regressions were run to assess the relationship among independent variables and dependent variable. First, data was organized and summarized in accordance to study objectives. Secondly, description of the summary sheets in order to produce a preliminary report was done. Data was collected through focus group discussion, questionnaire and informant interview schedule and were presented as per respective thematic areas.

Out of the 273 questionnaires administered to inmates, 233 were dully filled giving a response rate of $85 \%$. Out of 108 prison officers targeted for focus group discussion, 99 were available and fully took part in the discussion, representing a response rate of $92 \%$, while out of 4 officers in-charge and theirs deputies reached out for interview were all available and participated giving a response rate of $100 \%$ and therefore all these response were regarded as the responsive instrument for subsequent analysis. This was in line with the views of Cooper and Schiendler (2005) who observes that $75 \%$ and above response rate is reasonable enough for statistical generalization. In order to understand the characteristics of prisoners category respondents, the researcher was dealing with in the study, their background information was necessary, especially in relation to prison facilities in which the respondents were held. The study sought to elicit first information from the respondents on distribution by gender, age, number of years imprisoned, academic qualification section deployed. These are further discussed in the following sub sequent sub-themes. The demographic characteristics of the prisoners that were considered by the study were facilities, gender, age group, level of education, length of jail term in the prison facility. Distribution by facility was important to check capacity or whether the facilities were overstretched or if respondents were evenly distributed across the facilities under consideration. Distribution of respondents by gender was done to ascertain if health related reforms was in tandem with the specific needs of respondents and that none of the two genders was given preferential consideration in the selection of the respondents. Distribution of respondents by age group was done to ascertain that respondents held were of majority age and the minors were held in institutions where health related reforms were tailor made to suit their needs. Distribution of respondents by length of jail term in the prison facility was done to indicate how long the respondents had stayed in the facility to be able to see the reforms overtime and whether the health related reforms were designed to suit short and long sentence inmate needs. The distribution further indicated inter institution transfer which was necessary to check the variation on implementation as per facility. 
Table 4.1: Demographic characteristics of the prisoners $(n=233)$

\begin{tabular}{lc}
\hline Gender & n(f) frequency \\
Male & 216 \\
Female & 17 \\
Total & $\mathbf{2 3 3}$
\end{tabular}

(\%)Percentage

92.70

100

\author{
Age (years) \\ Below 18 \\ 18-25 \\ $26-35$ \\ $36-45$ \\ Over 46 years \\ Total \\ Length of jail term \\ Less than 5 yrs. \\ $6-10$ yrs. \\ $10-20$ yrs. \\ More than $21 \mathrm{yrs}$ \\ Total \\ Highest academic qualification \\ Primary school level \\ O Level \\ Diploma and above \\ Higher Diploma \\ Degree \\ Post graduate \\ Total
}

$\begin{array}{cc}3 & 1.28 \\ 78 & 33.47 \\ 109 & 46.78 \\ 30 & 12.87 \\ 13 & 5.57 \\ \mathbf{2 3 3} & \mathbf{1 0 0} \\ & \\ 139 & 59.65 \\ 57 & 24.46 \\ 30 & 12.87 \\ 7 & 3.00 \\ \mathbf{2 3 3} & \mathbf{1 0 0}\end{array}$

160160

68.66

$\begin{array}{rc}160 & 68.66 \\ 40 & 17.16 \\ 12 & 5.15 \\ 9 & 3.86 \\ 9 & 3.86 \\ 3 & 1.28 \\ \mathbf{2 3 3} & \mathbf{1 0 0}\end{array}$

\title{
4.1.1Change of behavior of inmates in correctional facilities
}

Change of behavior of inmate in correctional facilities was identified in the current study as the dependent variable. Re-offending, recidivism, jail breaks, riots and unruly habits as well as escapes while in prisons were identified as indicators of change of behavior of inmate in correctional facilities. Respondents were given items rated on a five-point Likert scale ranging from strongly agree (SA); agree (A); moderately agree (MA); disagree (DA); strongly disagree (SDA) from which to choose relating to each indicator. The findings were presented in Table 4.4 .

The research findings indicated that majority of the respondents disagreed that the number of riots by inmates has increased since the prison reforms were initiated in $2002(64.37 \%)$, were in disagreement that the number of unruly behavior cases has increased since the prison reforms were initiated in $2002(67.38 \%)$, while majority of the respondents agreed that the number re-offending within prison has been satisfactorily addressed $(68.66 \%)$, most respondents agreed only moderately. Majority of respondents further agreed that timely reporting of cases emanating from prison has improved satisfactorily $(80.67 \%)$ with bigger response being moderate however majority were in disagreement that jailbreaks has increased since inception of reforms in prisons $(67.37 \%)$. A small proportion of respondents agreed that the number of inmates who have escaped from prison have increased in the last ten years $(16.73 \%)$, while only half of respondents $(50.63 \%)$ disagreed that the number of inmate facing multiples cases in court has increased drastically with almost similar sentiments on whether the number of inmates who have committed offence again after release have increased drastically $(56.65 \%)$ were in disagreement. Regarding whether frequency of fights within prisons has significantly increased since the prison reforms were initiated in 2002 most of the respondents were in disagreement (55.64\%), and majority agreed that death cases resulting from riots has significantly reduced $(69.95 \%)$, most respondents were in agreement that the general living conditions in prisons has satisfactorily improved $(73.80 \%)$. Regarding change of behavior of inmates, there were varied responses with a big group of respondent agreeing only to a moderate extent in almost all items. This implies that there are several issues that need to be addressed to enable facilities to be able to impact positively on inmates behavior change underlying issues. 
Table 4.2. Means and standard deviation for inmates change of behavior in correctional facilities

\begin{tabular}{|c|c|c|c|c|c|}
\hline Statement & $\mathbf{N}$ & $\underset{\mathbf{n}}{\mathbf{M i}}$ & $\begin{array}{c}\mathbf{M a} \\
\mathbf{x}\end{array}$ & Mean & $\begin{array}{c}\text { Std. } \\
\text { Deviatio } \\
\text { n } \\
\end{array}$ \\
\hline $\begin{array}{l}\text { The number of riots by inmates has increased since the prison reforms } \\
\text { were initiated in } 2002\end{array}$ & 233 & 1 & 5 & 2.13 & 1.242 \\
\hline $\begin{array}{l}\text { The number of unruly behavior cases has satisfactory increased since } \\
\text { the prison reform were initiated in } 2002\end{array}$ & 233 & 1 & 5 & 2.24 & 1.187 \\
\hline $\begin{array}{l}\text { The number of re-offending within prison has been satisfactorily } \\
\text { addressed }\end{array}$ & 233 & 1 & 5 & 3.07 & 1.065 \\
\hline $\begin{array}{l}\text { Timely reporting of case emanating from prison has improved } \\
\text { satisfactory }\end{array}$ & 233 & 1 & 5 & 3.30 & 1.089 \\
\hline $\begin{array}{l}\text { Jailbreaks has increased since inception of reforms in prison for the } \\
\text { last ten years }\end{array}$ & 233 & 1 & 5 & 2.20 & 1.308 \\
\hline $\begin{array}{l}\text { The number of prison who have escaped from prison have increased } \\
\text { satisfactory since the last ten years }\end{array}$ & 233 & 1 & 5 & 2.18 & 1.304 \\
\hline $\begin{array}{l}\text { The number of inmate facing multiple cases in court have increased } \\
\text { drastically }\end{array}$ & 233 & 1 & 5 & 2.57 & 1.116 \\
\hline $\begin{array}{l}\text { The number of inmate who have committed offence again after } \\
\text { release have increased }\end{array}$ & 233 & 1 & 5 & 2.39 & 1.255 \\
\hline The death cases resulting from riots has significantly reduced & 233 & 1 & 5 & 3.66 & 1.308 \\
\hline $\begin{array}{l}\text { The number of fight within the prison has increased since the prison } \\
\text { reform were initiated in } 2002\end{array}$ & 233 & 1 & 5 & 2.19 & 1.074 \\
\hline The general living conditions in prisons has improved & 233 & 1 & 5 & 3.53 & 1.390 \\
\hline Composite mean & 233 & 1 & 5 & 2.68 & 1.219 \\
\hline
\end{tabular}

The research findings in table 4.5 showed that the mean score for the indicators used to measure inmates change of behavior was 2.68 and standard deviation of 1.219 . The study revealed a moderate agreement with the sentiment in each question item $\mathrm{M}(=2.68)$. Based on individual questionnaires item responses, the results showed that to a great extent, respondents disagreed the number of riots by inmates has increased since the prison reforms were initiated in $2002(\mathrm{M}=2.13, \mathrm{SD}=1.242)$. The number of unruly behavior cases has increased since the prison reform were initiated in $2002(\mathrm{M}=2.24, \mathrm{SD}=1.187)$. The number of re-offending within prison has been satisfactorily addressed( $M=3.07, \mathrm{SD}=1.065)$. Timely reporting of case emanating from prison has improved satisfactory $(\mathrm{M}=3.30, \mathrm{SD}=1.089)$. Jailbreaks has increased since inception of reforms in prison for the last ten years $(\mathrm{M}=2.20, \mathrm{SD}=1.308)$. The number of prison who have escaped from prison have increased satisfactory since the last ten years $(\mathrm{M}=2.18, \mathrm{SD}=1.304)$.

The number of inmate facing multiple cases in court have increased drastically $(\mathrm{M}=2.57, \mathrm{SD}=1.116)$. To a moderate extent, the number of inmates who have committed offence again after release have increased drastically $(\mathrm{M}=2.39, \mathrm{SD}=1.255)$. Agreed that death cases resulting from riots has significantly reduced $(\mathrm{M}=3.66$, $\mathrm{SD}=1.308)$. Disagreed that the number of fight within the prison has significantly increased since the prison reform were initiated in $2002(\mathrm{M}=2.19, \mathrm{SD}=1.074)$. Agreed that the general living conditions in prisons has satisfactorily improved $(\mathrm{M}=3.53, \mathrm{SD}=1.390)$.

\subsubsection{Descriptive analysis of healthrelated reforms on change of behavior of inmates in correctional} facilities.

This section presented data analysis and findings on the indicators of health related reforms, The research findings on Table 4.2 indicated that majority of the respondents agreed that facilities had functional ambulances to provide services when needed in correctional facilities $(73.38 \%)$, were in agreement that patients have access to the ambulance when they need them $(46.78 \%)$, while nearly half of the respondents were in disagreement that awareness about best practice and usage of ambulance is created often $(47.63 \%)$, while respondents further agreed that services offered by clinical officers at the prison health centers are satisfactory (46.78\%), however respondents were in agreement that services offered by nurses at the prison health centers are satisfactory $(45.06 \%)$. A small proportion of respondents agreed that the medic are available when required to attend to a staff, inmate, or any stake holder $(11.58 \%)$, while only $(46.77 .9 \%)$ agreed that the staff and inmates have access to specialized Medicare with similar sentiments on whether awareness is created to staff and inmates about specialized Medicare in prisons (34.75\%) were in agreement. Regarding whether there are dispensaries or health centers at the institution, most of the respondents were in agreement $(64.8 \%)$, and minority agreed that the state of health facilities is humane (39.91\%), respondents were in disagreement that drugs are available when required by staff, or inmates (46.34\%). Regarding health reforms, there were mixed opinions on the items with some skewed to disagreement and others in agreement with a big number of respondents remaining neutral on most items. This implies that health reform has several issues that need to be clearly addressed to meet health care 
expectations of beneficiaries.

\subsubsection{Means and standard deviation for health related reform}

Items were rated as strongly agree (5) to strongly disagree (1). A score of $\leq 1.5$ was interpreted to mean strongly disagree, while a score 1.5 to $\leq 2.5$ implied that the respondents disagrees and 2.5 to $\leq 3.5$ was interpreted to mean that the respondent was neutral. A mean score of $3.5 \leq 4.5$ was interpreted to mean that respondents agree while a score of $\geq 4.5$ was interpreted to mean strongly agree. A standard deviation of $\leq 1$ was interpreted to mean that respondents had a consensus in the rating of the statement while a standard deviation greater than 1 was interpreted to mean that the respondents differed in their perception about the statement.

Table 4.3: Means and standard deviation for health related reforms

\begin{tabular}{|c|c|c|c|c|c|}
\hline Statement & $\mathbf{N}$ & Min & Max & Mean & $\begin{array}{l}\text { Std. } \\
\text { Deviation }\end{array}$ \\
\hline The facility has functional ambulance & 233 & 1 & 5 & 3.78 & 1.054 \\
\hline Patients have access to ambulance when & 233 & 1 & 5 & 3.12 & 1.340 \\
\hline $\begin{array}{l}\text { The services offered by clinical officers at the } \\
\text { prison facilities are satisfactory }\end{array}$ & 233 & 1 & 5 & 3.82 & 1.214 \\
\hline $\begin{array}{l}\text { Awareness about best practice and usage of } \\
\text { ambulance is created }\end{array}$ & 233 & 1 & 5 & 2.67 & 1.283 \\
\hline Service delivery by the nurses is satisfactory & 233 & 1 & 5 & 3.14 & 1.367 \\
\hline $\begin{array}{l}\text { The medic are available when required to attend a } \\
\text { inmate }\end{array}$ & 233 & 1 & 5 & 2.32 & 1.001 \\
\hline $\begin{array}{l}\text { Awareness is created about specialized Medicare } \\
\text { by prisons }\end{array}$ & 233 & 1 & 5 & 2.91 & 1.278 \\
\hline $\begin{array}{l}\text { There are dispensaries or health centers at the } \\
\text { institution }\end{array}$ & 233 & 1 & 5 & 3.68 & 1.116 \\
\hline State of health facilities is humane & 233 & 1 & 5 & 3.02 & 1.320 \\
\hline Drugs are available when required & 233 & 1 & 5 & 2.77 & 1.490 \\
\hline Inmate have access to specialized Medicare & 233 & 1 & 5 & 2.84 & 1.227 \\
\hline Composite mean & 233 & 1 & 5 & 3.097 & 1.245 \\
\hline
\end{tabular}

The research findings in Table 4.3 showed that the mean score for the eleven statements for health related reform was 3.097 and standard deviation of 1.245. From individual items' mean and standard deviation, it was clear that the respondents either agreed or disagreed with the state of health related reforms in the facilities. Agreed that the facility has functional ambulance $(\mathrm{M}=3.78, \mathrm{SD}=1.054)$, agreed that patients have access to ambulance when they need them $(\mathrm{M}=3.12, \mathrm{SD}=1.340)$, agreed that services offered by clinical officers at the prison facilities are satisfactory $(\mathrm{M}=3.82, \mathrm{SD}=1.214)$, were neutral that awareness about best practice and usage of ambulance is created often $(\mathrm{M}=2.67, \mathrm{SD}=1.283)$. Were in agreement that service delivery by the nurses is satisfactory $(\mathrm{M}=3.14, \mathrm{SD}=1.367)$, disagreed that there is adequate medics available when required to attend inmate $(\mathrm{M}=2.32, \mathrm{SD}=1.001)$, and also moderately agreed that awareness is created about specialized Medicare by prisons $(\mathrm{M}=2.91, \mathrm{SD}=1.278)$ and agreed that there exist adequate dispensaries or health centers at the institution $(\mathrm{M}=3.68, \mathrm{SD}=1.116)$. They moderately agreed that state of health facilities is humane $(\mathrm{M}=3.02$, $\mathrm{SD}=1.320)$, were in moderate agreement that drugs are available when required by inmates $(\mathrm{M}=2.77, \mathrm{SD}=1.490)$ and also agreed moderately that inmate have access to specialized medicare $(\mathrm{M}=2.84, \mathrm{SD}=1.227)$.

The overall mean and standard deviation $(\mathrm{M}=3.097, \mathrm{SD}=1.245)$, implied that the respondents had responses that were spread around the mean. They either agreed moderately or disagreed regarding the issues raised through the questionnaire, hence their responses concentrated around the mean. These research findings implied that health related reforms is a major factor in correctional facilities reforms that need to be wholly addressed in order to have great impact on change of behavior of inmates in correctional facilities.

\subsubsection{Correlational analysis of health related reforms and change of behavior of inmates in correctional} facilities

This section presents the results of the correlation analysis of study variables using Pearson's product-moment correlation. The Pearson product-moment correlation coefficient is a measure of the strength of a linear association between two variables and is denoted by $r$. Correlational analysis using Pearson's Product Moment technique was done to determine the relationship between health related reforms and change of behavior of inmates. The purpose was to identify the strength and direction of the association between health related reforms and change of behavior of inmates. Values of correlation coefficient range from- 1 and +1 . A correlation coefficient of +1 indicates that the two variables are perfectly and positively related linearly. A value of 0 indicates that there is no association between the two variables. A value greater than 0 indicates a positive association; that is, as the value of one variable increases, so does the value of the other variable. A value less than 0 indicates a negative association; that is, as the value of one variable increases, the value of the other 
variable decreases. A value of 1 indicates perfect positive correlation implying that an increase or decrease in one variable is followed by a proportional increase/decrease in the other variable while a value of -1 indicate perfect negative correlation which imply that an increase in one variable is followed by a proportional decrease in the other variable (Cooper \& Schindler, 2003). Correlation coefficient (r) range of between 0.81 to 1.0 is very strong; from 0.61 to 8.80 is strong; from $0.41-0.60$ is moderate; from $0.21-0.40$ is weak and from 0.00 to 0.20 indicate no relationship (Hair et al., 20 06).

The stronger the association of the two variables, the closer the Pearson correlation coefficient, $r$, will be to either +1 or -1 depending on whether the relationship is positive or negative, respectively (Cooper \& Schindler, 2003). According to Sekaran (1992), the Pearson's correlation is used if the variables of the study are measured using either interval or ratio scales. Correlation results are reported at a significance level of 0.05 and 0.01 .

Table 4.10: Correlation table for health related reforms and change of behavior of inmates

\begin{tabular}{lll}
\hline & & Health Reform \\
\hline Change of Behavior of Inmates & Pearson correlation & $0.428^{* * *}$ \\
& Sig (2-tailed) & 0.000 \\
& $\mathrm{~N}$ & 233 \\
\hline
\end{tabular}

**correlation is significant at the 0.01 level (2-tailed)

The correlation results in table 4.10 indicated a correlation of 0.428 and a significance of 0.000 (two tailed test), which imply a positive and significant coefficient. The results further showed a moderate and significant relationship between health reforms and change of behavior of inmates $(r=0.428, P$-value $<0.000)$. Therefore health related reforms can be said to have a positive and significant relationship with change of behavior of inmates in correctional facilities. The results indicated a correlation of 0.428 and a significance of 0.000 (two tailed test), which imply a positive and significant coefficient. The result further indicated a moderate and significant relationship $(\mathrm{r}=0.428$, P-value $<0.000)$ with change of behavior of inmates as summarized in Table 4.10.

Hypothesis test

To test for hypothesis $\mathbf{H}_{0}$ : There is no significant relationship between health related reforms and change of behavior of inmates in correctional facilities in Kenya.

Table 4.4: Regression results of influence of health related reforms on change of inmates behavior in correctional facilities

\begin{tabular}{lccccc}
\hline Model & \multicolumn{2}{c}{$\begin{array}{c}\text { Unstandardized } \\
\text { Coefficients }\end{array}$} & \multicolumn{2}{c}{$\begin{array}{c}\text { Standardized } \\
\text { Coefficients }\end{array}$} & t \\
\hline B & Std.Error & Beta & & P-Value \\
Constant & 2.506 & 0.139 & & 12.182 & 0.000 \\
Health related reforms & 0.307 & 0.051 & 0.429 & 5.189 & 0.000
\end{tabular}

Predictors: (Constant) Health related reforms

Dependent Variable: Change of Behavior of Inmates

\author{
$\mathrm{R}=\mathbf{0 . 4 2 9}$ \\ R square $=0.184$ \\ $t=5.189$ at level of significance $p=0.000<0.05$
}

The study findings in Table 4.4 showed that $r$ was equal to 0.429 indicating that health related reforms had a moderately strong influence on change of inmate's behavior in correctional facilities. The value of $\mathrm{r}^{2}$ was 0.184 , indicating the health reforms explained $18.4 \%$ of the variation in change of inmate's behavior in correctional facilities in Nairobi county, Kenya. The $\beta$ coefficient of health reforms was statistically significant 0.429 , indicated that significant influence on change on inmates behavior in correctional facilities $(\beta=0.429, \mathrm{t}=5.189$. $\mathrm{p}=0.000<0.05)$. The $\beta$ value implied that one unit change in inmates behavior change was associated with $42.9 \%$ changes in health reform.

The overall $\mathrm{t}=5.189$ with $\mathrm{p}=0.000<0.05)$ suggested that there was a statistically significant relationship between health related reforms and change of behavior of inmates in correctional facilities. Based on the research findings the null hypothesis which stated that there is no significant relationship between health reforms and the change of behavior of inmates in correctional facilities in Kenya was rejected, and alternative hypothesis accepted Using the statistical findings, the regression model was substituted as follow

$\mathrm{Y}=2.506+0.429 \mathrm{X}+\varepsilon$ 


\subsection{Recommendations}

Considering the government of Kenya as well as many other developing countries were gearing towards implementation of reforms that will ensure correctional facilities are run in humane manner putting into consideration international standards. This study has implication to government implication agencies and especially ministry of interior and coordination of national government in which the prison department is placed. Study findings indicated that each of the health related reforms variables had an influence on change of behavior of inmates in correctional facilities in Kenya.

\section{REFERENCES}

Abuyeka, R. O. (2014). Inmates' and Warders' Perception of Prison Reforms in Kenya: A Case of EldoretGk Prison, in Eldoret, Kenya. Public Policy and Administration Research, Vol. 4(11),17-20.

Achu, A., Owan, E. J., \& Ekok, O. C. (2013).The impact of prison reform on the welfare of Inmates. A case study of Afonkong prisonCalabar, Cross River State, Nigeria. Global Journal of Human social science sociology and culture, 13,1-7.

Adler, M., \& Longhurst, B. (1994).Discourse, power and justice: Towards a new sociology ofimprisonment.Routledge. London.

Adlerstein, D. M. (2001).In Need of Correction: The "Iron Triangle" of the Prison Litigation Reform Act. Columbia Law Review, Vol. 101(7), pp. 1681-1708.

Alexopoulos, E. C. (2010). Introduction to Multivariate Regression Analysis.Quarterly Medical Journal,14(1), $23-28$.

Bayens, G. J., Williams, J. J., \& Smykla, J. O. (2012).Jail type and inmate behavior.A longitudinal analysis. Federal Probation, 61(3), 54-59

Becker, K. L. (2003). HIV and AIDS in prisons. British Medical Association Foundation for AIDS. London: $2,(6), 35-41$.

Behn, R. (2003).Why measureperformance?Differentpurposerequiresdifferent measure http://www.coe.int/t/dghl/cooperation/cepej/thematiques/Measuring_perf/Whymeasureperformance [online]

Behn, R. (2003). Why measure performance? Different purposes requiredifferentmeasures. Public Administration Review, 63, 586 -606.

Bhalerao C. N. (2000). Urgent Need for Prison Reforms.Economic and Political Weekly, Vol. 35(10), p. $746-751$.

Brown, A. (1993). Economic and qualitative aspects of prison privatization in Queensland, in Moyle, P. (Ed.), Private Prisons and Police, Pluto Press, Sydney, NSW

Brown, E. (2012). Diet, exercise, obesity in prisons worldwide, Los Angeles Times

Bryce, W. (2003). Evaluating Government Regulations. A Journal of Policy Analysis and Reform, Vol. 10, 4, 325-339

Bryman, A., \& Bell, E. (2003). Business Research Methods. New York: Oxford University Press

Bryman, A., \& Cramer, D. (1997). Quantitative Data Analysis with SPSS for Windows: A guide for social scientists. London: Routledge.

Creswell, J.W., \& Clarke, V. L. (2007). Designing and Conducting Mixed Methods Research. Los Angeles: Sage.

Denzin, N. (2010). Moments, mixed methods, and paradigm dialogs.Qualitative Inquiry, 16, 419-427.

DiPrete, T. A. \& Eirich, G. M. (2006). Cumulative advantage as a mechanism for inequality: a' review of theoretical and empirical developments, Annual Review of Sociology, Vol. 32, pp. 271-97

Isaac, S., \& Michael, W. (2002). Handbook in Research and Evaluation: For Education and the Behavioral Sciences. San Diego: Ed. ITS Publishers

KNCHR (2010). Integration of human rights provisions in African prisons. Kampala Uganda

Koech, J. K. (2009). The Effect of Open Door Policy on Service Delivery in Prisons, Independent Paper, Day Star University

Kothari C. R. (2005). Research Methodology: Methods and Technology, New Age International Publishers

Nachmias, C. F. \& Nachmias, D. (1996). Research Methods, Departmental Heads in Social Sciences. (5thEd.) London: St. Martin's Press. 